\title{
Six New Factors of Fermat Numbers
}

\section{By Gary B. Gostin and Philip B. McLaughlin, Jr.}

\begin{abstract}
A new prime factor is given for each of the Fermat numbers $F_{29,} F_{36,}, F_{99}, F_{147}$, $F_{150,}$ and $F_{201}$. A summary of search limits and recent results is included.
\end{abstract}

In the past few years several investigators [1]-[11] have found many new prime factors of the Fermat numbers $F_{m}=2^{2^{m}}+1$. The increased availability and speed of computing equipment have allowed the search limits for factors of these numbers to be extended significantly. In tables below, we include the known search limits and all of the results that have been obtained since H. C. Williams' 1978 paper [12] was published.

Using the method outlined by Hallyburton and Brillhart [8], we tested integers of the form $k \cdot 2^{n}+1, k$ odd, as factors of the Fermat numbers $F_{m}, 9<m<n-2$, for the ranges shown in Table 1. During our search the following new prime factors were found:

$$
\begin{gathered}
1120049 \cdot 2^{31}+1 \mid F_{29} \\
3759613 \cdot 2^{38}+1 \mid F_{36} \\
16233 \cdot 2^{104}+1 \mid F_{99} \\
3125 \cdot 2^{149}+1 \mid F_{147} \\
5439 \cdot 2^{154}+1 \mid F_{150} \\
4845 \cdot 2^{204}+1 \mid F_{201}
\end{gathered}
$$

The factors of $F_{36}$ and $F_{150}$ are the second known for these numbers.

TABLE 1

Intervals covered by the authors for $k \cdot 2^{n}+1, k$ odd.

\begin{tabular}{cl}
\hline$n$ & \\
\hline 25 & $2^{22}<k<2^{23}$ \\
$26-27$ & $2^{21}<k<2^{23}$ \\
28 & $2^{20}<k<2^{23}$ \\
$29-40$ & $10^{6}<k<2^{23}$ \\
41 & $10^{6}<k<2^{22}$ \\
$42-55$ & $10^{6}<k<2^{21}$ \\
$56-102$ & $10^{6}<k<2^{20}$ \\
103 & $10^{3}<k<2^{19}$ \\
\hline
\end{tabular}

\begin{tabular}{cl}
\hline$n$ & \\
\hline $104-113$ & $10^{3}<k<2^{18}$ \\
$114-135$ & $10^{3}<k<2^{17}$ \\
$136-143$ & $10^{3}<k<2^{16}$ \\
$144-176$ & $10^{3}<k<2^{15}$ \\
177 & $10^{3}<k<2^{14}$ \\
$178-242$ & $10^{3}<k<2^{13}$ \\
$243-245$ & $10^{3}<k<2^{255-n}$ \\
\hline
\end{tabular}

Received December 17, 1980; revised March 19, 1981.

1980 Mathematics Subject Classification. Primary 10A25, 10A40; Secondary 10-04.

Key words and phrases. Fermat numbers, factorization. 
Note. We did not test the range $2^{15}<k<2^{16}$ for $n=120,128$, or 136 . These intervals were covered by H. Suyama. See Table 2 .

The first-named author programmed the method in assembly language on a Motorola MC6800 microprocessor. To test a trial divisor $d_{k}=k \cdot 2^{n}+1$ as a factor, the residue $2^{2^{m}}\left(\bmod d_{k}\right)$ was calculated for $9 \leqslant m<n-2$. The number of trial divisors were reduced by sieving through a set of modulo $p_{i}$ counters, where $p_{i}$ is the $i$ th prime, $2 \leqslant i \leqslant 31$. Counter $i$, upon counting down to zero, would signify division of $d_{k}$ by $p_{i}$, and the counter would be reset to $p_{i}$. This program has been running, with only brief interruptions, since September, 1979.

The second-named author programmed the method in HPL (a machine-specific language) on a Hewlett-Packard 9825 desktop computer. To reduce the number of trial divisors $d_{k}$, the values of $k$ were sieved. This consisted of computing and storing, for a given value of $n$, the quantities $c_{i}=-\left(\left(p_{i}+1\right) / 2\right)^{n}\left(\bmod p_{i}\right)$, where $p_{i}$ is the $i$ th prime. It is easy to show that $p_{i}$ divides $d_{k}=k \cdot 2^{n}+1$ if, and only if, $k$ $\left(\bmod p_{i}\right)=c_{i}$. Thus only those $d_{k}$ 's for which $k\left(\bmod p_{i}\right) \neq c_{i}, 2<i<L$, where $L$ was variable up to 1300 , were tested as possible factors. This program has been running almost continuously since January, 1980.

TABLE 2

Search limits for $k \cdot 2^{n}+1, k$ odd, $3<k<L_{k}$.

\begin{tabular}{|c|c|c|}
\hline$n$ & $L_{k}$ & Recent investigators \\
\hline $11-23$ & $2^{47-n}$ & Montgomery $(n<15)$ [9], Gostin [7] \\
\hline 24 & $2^{24}$ & Hallyburton \& Brillhart [8] \\
\hline $25-40$ & $2^{23}$ & \multirow{3}{*}{ Baillie $\left(k<10^{6}\right)[2],[3]$} \\
\hline 41 & $2^{22}$ & \\
\hline $42-55$ & $2^{21}$ & \\
\hline $56-102$ & $2^{20}$ & \multirow{8}{*}{ Suyama $(k<f)[10],[11]$} \\
\hline 103 & $2^{19}$ & \\
\hline $104-113$ & $2^{18}$ & \\
\hline $114-135$ & $2^{17}$ & \\
\hline $136-144$ & $2^{16}$ & \\
\hline $145-177$ & $\max \left(2^{15}, f\right)^{*}$ & \\
\hline $178-243$ & $\max \left(2^{13}, f\right)$ & \\
\hline $244-418$ & $\max (1000, f))^{\prime}$ & \\
\hline $419-600$ & 1000 & \multirow{3}{*}{ Baillie [2], [3] } \\
\hline $601-1000$ & 280 & \\
\hline $1001-1500$ & 150 & \\
\hline $1501-4000$ & 30 & Cormack \& Williams [6] \\
\hline
\end{tabular}

* $f=2^{16-r}$, where $r=n(\bmod 8), 0<r<7$.

Note. The exponent $n$ has been searched up to 8000 for $k=7,17,27$, and 29, and up to 10000 for $k=5[6]$. 
It is interesting to note that full multiple-precision division is not necessary when computing residues modulo $d_{k}$. Instead, given the previous residue $2^{2^{i-1}}\left(\bmod d_{k}\right)=$ $r_{i-1}, 0<r_{i-1}<d_{k}$, calculate $r_{i-1}^{2}$, and then compute $q_{i}$ and $r_{i}^{\prime}$, where $r_{i-1}^{2}=$ $q_{i}\left(k \cdot 2^{n}\right)+r_{i}^{\prime}, 0<r_{i}^{\prime}<k \cdot 2^{n}$. When using a base-2 number system, this division can be reduced to division by $k$ and some shift operations. The next residue is now easily obtained: $r_{i}=r_{i}^{\prime}-q_{i}\left(\bmod d_{k}\right)$. Moreover, $-k \cdot 2^{n}<r_{i}^{\prime}-q_{i}<k \cdot 2^{n}$, so that at most a single addition of $d_{k}$ is required to force $0<r_{i}<d_{k}$.

TABLe 3

Recent factors of the form $k \cdot 2^{n}+1$ of $F_{m}$, and related results.

\begin{tabular}{rrrrl}
\hline$m$ & $k$ & $n$ & Date & Discoverer \\
\hline 8 & 604944512477 & 11 & 1980 & R. P. Brent [4] \\
8 & $p^{*}$ & & 1980 & R. P. Brent, H. C. Williams [5] \\
11 & $c^{* *}$ & & 1979 & S. S. Wagstaff (see [7]) \\
12 & $c$ & & 1979 & S. S. Wagstaff (see [7]) \\
13 & $c$ & & 1979 & S. S. Wagstaff (see [7]) \\
17 & 59251857 & 19 & 1978 & G. B. Gostin [7] \\
29 & 1120049 & 31 & 1980 & G. B. Gostin, P. B. McLaughlin \\
36 & 3759613 & 38 & 1981 & G. B. Gostin, P. B. McLaughlin \\
93 & 92341 & 96 & 1979 & R. Baillie [2] \\
99 & 16233 & 104 & 1979 & G. B. Gostin, P. B. McLaughlin, \\
& & & & H. Suyama [10] \\
147 & 3125 & 149 & 1979 & G. B. Gostin, P. B. McLaughlin \\
150 & 5439 & 154 & 1980 & G. B. Gostin, P. B. McLaughlin, \\
& & & & H. Suyama [10] \\
201 & 4845 & 204 & 1980 & G. B. Gostin, P. B. McLaughlin \\
215 & 32111 & 217 & 1980 & H. Suyama [10] \\
255 & 629 & 257 & 1979 & R. Baillie [2] \\
287 & 5915 & 289 & 1980 & H. Suyama [10] \\
298 & 247 & 302 & 1979 & R. Baillie [2] \\
329 & 1211 & 333 & 1981 & H. Suyama [10] \\
416 & 8619 & 418 & 1981 & H. Suyama [11] \\
544 & 225 & 547 & 1979 & R. Baillie [2] \\
692 & 717 & 695 & 1979 & A. O. L. Atkin, N. W. Rickert [1] \\
1551 & 291 & 1553 & 1979 & A. O. L. Atkin, N. W. Rickert [1] \\
2023 & 29 & 2027 & 1979 & Atkin, Rickert, Cormack, Williams [1], [6] \\
2456 & 85 & 2458 & 1979 & A. O. L. Atkin, N. W. Rickert [1] \\
3310 & 5 & 3313 & 1979 & Atkin, Rickert, Cormack, Williams [1], [6] \\
4724 & 29 & 4727 & 1979 & G. V. Cormack, H. C. Williams [6] \\
6537 & 17 & 6539 & 1979 & G. V. Cormack, H. C. Williams [6] \\
\hline & & & &
\end{tabular}

* cofactor is prime.

** cofactor is composite.

The time required to cover the different intervals in Table 1 varied considerably. To test a single trial divisor on the 9825 took about 4 seconds for $n$ near 40,25 
seconds for $n$ near 100 , and about 150 seconds for $n$ near 175 . For any particular $n$, the time spent searching ranged anywhere from 50-75 hours each for $n$ in the range 144-176, to up to 10 days or more for $n$ in the range 25-40. The two machines used had quite similar computation speeds.

In Table 2 above, we give a summary of the search limits known to us for factors of Fermat numbers. The names of recent researchers other than the authors, some of whose work has not been published previously, are included.

Samuel Wagstaff has informed us that Hiromi Suyama, working independently in Japan, has also discovered the factors of $F_{99}$ and $F_{150}$ mentioned above. In addition, he has found four other new factors of Fermat numbers. These, as well as all of the other factors and related results that have been published since Williams' paper [12], are listed in Table 3. We understand that a full account of Suyama's work will be published elsewhere.

The reader should note an error in [12]. In Table 4 on p. 135, the fifth entry should read "30 12758933 ", not "30 12758930 ".

There are now 85 prime factors known for 71 composite Fermat numbers. In addition, one other Fermat number and five cofactors are known to be composite.

Finally, there is a well-known conjecture that the Fermat numbers are squarefree. We tested all of the known prime factors $p$ of $F_{m}, 9<m<2456$, to determine if $p^{2}$ divided $F_{m}$. No such factor was found. In each case, the residue $R=2^{2^{m}}$ $\left(\bmod p^{2}\right)$ was subsequently divided by $p$ to verify that $R(\bmod p)=-1$. The test for $85 \cdot 2^{2458}+1$ took about 165 hours. To our knowledge, the three largest factors in Table 3 have not been so tested.

Acknowledgement. The authors wish to thank Robert Baillie, Peter Montgomery, and Hiromi Suyama for their contributions to this paper, and Samuel Wagstaff for supplying helpful suggestions and information.

Note Added in Proof. We have extended our search to the following limits:

\begin{tabular}{cc}
\hline$n$ & $L_{k}$ \\
\hline 22 & 35750886 \\
$25-27$ & $2^{24}$ \\
$145-181$ & $2^{16}$ \\
$247-702$ & $2^{10}$ \\
$703-819$ & $2^{9}$ \\
\hline
\end{tabular}

Intervals already covered were bypassed. In addition, Hiromi Suyama has searched $419<n<575$ for $k<f$ (see Table 2). No new factors were discovered.

571 Havencrest Lane

Coppell, Texas 75019

919 Northwood \#7604

Baytown, Texas 77521

1. A. O. L. Atkin \& N. W. Rickert, "Some factors of Fermat numbers," Abstracts Amer. Math. Soc., v. 1, 1980, p. 211.

2. ROBert Baillie, "New primes of the form $k \cdot 2^{n}+1$," Math. Comp., v. 33, 1979, pp. 1333-1336.

3. Robert BaIllie, Personal communication via S. S. Wagstaff, Jr. 
4. R. P. BRENT, "Factorization of the eighth Fermat number," Abstracts Amer. Math. Soc., v. 1, 1980, p. 565 .

5. R. P. Brent \& J. M. Pollard, "Factorization of the eighth Fermat number," Math. Comp., v. 36, 1981, pp. 627-630.

6. G. V. Cormack \& H. C. Williams, "Some very large primes of the form $k \cdot 2^{n}+1$," Math. Comp., v. 35, 1980, pp. 1419-1421.

7. GARY B. Gostin, “A factor of $F_{17}$ ” Math. Comp., v. 35, 1980, pp. 975-976.

8. John C. Hallyburton, JR. \& John Brillhart, "Two new factors of Fermat numbers," Math. Comp., v. 29, 1975, pp. 109-112. For a correction, see Math. Comp., v. 30, 1976, p. 198.

9. Peter Montgomery, Personal communication.

10. Hiromi SUyama, "Searching for prime factors of Fermat numbers with a microcomputer," BIT (Japanese), v. 13, 1981, pp. 240-245.

11. Hiromi Suyama, Personal Communication; published with permission.

12. H. C. Williams, "Primality testing on a computer," Ars Combin., v. 5, 1978, pp. 127-185. 